ARTICLE

Received 26 Aug 2016 | Accepted 13 Jan 2017 | Published 24 Feb 2017

DOI: $10.1038 /$ ncomms14579

OPEN

\title{
An intelligent artificial throat with sound-sensing ability based on laser induced graphene
}

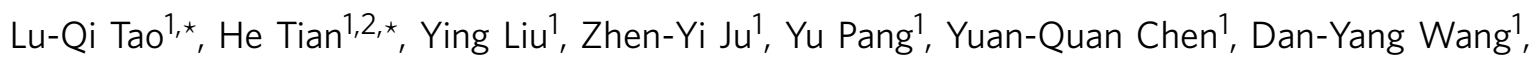
Xiang-Guang Tian ${ }^{1}$, Jun-Chao Yan ${ }^{1}$, Ning-Qin Deng ${ }^{1}$, Yi Yang $^{1} \&$ Tian-Ling Ren ${ }^{1}$

Traditional sound sources and sound detectors are usually independent and discrete in the human hearing range. To minimize the device size and integrate it with wearable electronics, there is an urgent requirement of realizing the functional integration of generating and detecting sound in a single device. Here we show an intelligent laser-induced graphene artificial throat, which can not only generate sound but also detect sound in a single device. More importantly, the intelligent artificial throat will significantly assist for the disabled, because the simple throat vibrations such as hum, cough and scream with different intensity or frequency from a mute person can be detected and converted into controllable sounds. Furthermore, the laser-induced graphene artificial throat has the advantage of one-step fabrication, high efficiency, excellent flexibility and low cost, and it will open practical applications in voice control, wearable electronics and many other areas.

\footnotetext{
${ }^{1}$ Institute of Microelectronics and Tsinghua National Laboratory for Information Science and Technology (TNList), Tsinghua University, Beijing 100084, China.

${ }^{2}$ Ming Hsieh Department of Electrical Engineering, University of Southern California, Los Angeles, California 90089, USA. * These authors contributed equally to this work. Correspondence and requests for materials should be addressed to Y.Y. (email: yiyang@tsinghua.edu.cn) or to T.-L.R.

(email: RenTL@tsinghua.edu.cn).
} 
O wing to diseases and unexpected accidents, a lot of people in this world are not able to speak out with languages. Some technologies have been developed to help them express themselves in other ways. For example, eye tracking technology is developed to convert the eye movements into language expressions. They can use their eyes to focus on their wanted characters ${ }^{1,2}$. However, these technologies are not widely applied due to their complexities and extreme costs. In fact, many mute people have the ability to produce some kind of sounds such as a cough, hum or scream, which do not have clear meaning to other people. It is of great significance to develop an easy-to-use artificial throat that can convert the sound with unclear meaning into controllable and precise sound in languages. It means that the artificial throat should have the capabilities of both detecting and generating sound. However, acoustic transducers that can achieve this usually work with narrow bandwidth in the ultrasound range ${ }^{3}$ or under water ${ }^{4,5}$. Traditional sound sources and detectors are usually isolated from each other and are discrete within the human hearing range. Besides, they have the drawbacks of resonant peak and poor flexibility, which are not suitable for wearable applications.

The research of nanomaterials and nanotechnologies are contributing to the development of novel acoustic devices based on new mechanisms. For example, thermoacoustic sound sources based on graphene $e^{6-12}$, carbon nanotube ${ }^{13,14}$, silver nanowires ${ }^{15}$ and indium tin oxide ${ }^{16}$ have attracted a lot of attentions due to their high transparency, low heat capacity, broad frequency and excellent flexibility. However, the complex fabrication process, low yield and high cost will restrict the commercial and practical applications of thermoacoustic sources. Except novel sound sources, some nanomaterial-based novel sound detectors ${ }^{17-22}$ have been developed based on the piezoresistive effect. These novel sound detectors have responses towards the movement of throat. However, the responses are indistinctive and there is still a long way to make them for practical applications. Most importantly, the different working mechanisms of thermoacoustic sound sources and piezoresistive sound detectors make it hard to realize the functional integration of the sound source and detector in a single device. On one hand, the subsistent sound detectors based on piezoresistive materials ${ }^{18,21-23}$ cannot work as sound sources. Most of these sensors were encapsulated in the elastic polymers such as Ecoflex $^{20}$ or polydimethylsiloxane ${ }^{22}$, which made it impossible to emit Joule heating into the air so that they cannot work as sound sources. The rest sensors ${ }^{18,21,23}$ have very high original resistances (up to $\mathrm{M} \Omega$ ); therefore, little Joule Heating could be generated. Previous reported sound detectors cannot work as sound sources; thus, these kinds of sound detectors can be replaced by conventional microphones. On the other hand, the thermal sound sources that were developed previously ${ }^{11}$ cannot work as sound detectors, because the gauge factor of laser scribed graphene is only 0.11 (ref. 24), which means it has a poor sensitivity and is hard to work as a sound detector; thus, these kinds of sound sources can be replaced by conventional speakers. However, none of the previously demonstrated nanomaterial-based acoustic device can have both soundemitting and -detecting ability in hearing range, and this kind of multifunctional device would not be replaced by any conventional devices. Nevertheless, the functional integration of generating and detecting hearable sound in a single device has not been explored until now.

It is known that laser scribing technology has been demonstrated as an efficient method to fabricate graphene-based devices $^{25-30}$. Some graphene devices based on laser scribing technology has been developed ${ }^{11,27}$, which shows advantages of large scale and customized pattern. However, the preparation of graphene oxide and the spin coating process still cause a waste of time. Lin et al. ${ }^{31}$ developed a method of converting polyimide (PI) into porous graphene films via one-step process. This method is further applied to develop supercapacitor energy storage devices ${ }^{32}$ and flexible strain sensors ${ }^{33,34}$. The high thermal conductivity and low heat capacity of laser induced graphene (LIG) is ideal for thermoacoustic sound sources. Besides, the porous structure will have a sensitive response towards weak vibrations, which is suitable for sound detection. Therefore, the simple and one-step fabrication will be suitable for the realization of this novel artificial throat.

Herein, we develop a one-step, wearable and low-cost LIG artificial throat by direct laser writing of PI and it shows good performance in generating and detecting sounds. This LIG artificial throat has completely different working mechanism compared with conventional acoustic transducers, which usually utilize piezoelectric effect and inverse piezoelectric effect ${ }^{35-37}$. When working as a sound source, LIG artificial throat can generate wide-band sound with frequency from $100 \mathrm{~Hz}$ to $40 \mathrm{kHz}$. A thinner LIG will produce higher sound pressure level (SPL). When working as a sound detector, LIG artificial throat shows unique responses towards different kinds of sounds and throat vibration modes. LIG can recognize cough, hum and scream with different tones and volumes. Besides, it also has the capability of recognizing words and sentences. Benefiting from its capability of generating and detecting sounds, the intelligent LIG artificial throat will significantly assist for the disabled. The throat vibration with different volume and frequency can be converted into controllable and predesigned sounds. Furthermore, the LIG artificial throat has the advantage of one-step process, high efficiency, excellent flexibility and low cost. The LIG can be acquired by using a portable and low-power laser platform, which will reduce the risk under operation. The LIG artificial throat has promising applications in the fields including voice control, wearable electronics and many others.

\section{Results}

Fabrication and characterization of LIG artificial throat. Laser direct writing technology promotes the fast growth of porous graphene, and a low-cost and portable laser platform is chosen in this work (Supplementary Fig. 1). Figure 1a shows the one-step process of the LIG. The PI film is located under the $450 \mathrm{~nm}$ laser and converted into LIG by direct irradiation of laser. The $X-Y$ directional motors control the movement of the laser so that a predesigned pattern can be irradiated at precise locations (see the Methods section for details). For instance, Tsinghua University's logo and a $6 \mathrm{~cm} \times 4 \mathrm{~cm}$ rectangle are imported into the computer control software, and the same patterns are generated on the PI film by converting PI into graphene (Supplementary Fig. 2). Here, a simple rectangle LIG is produced to work as an artificial throat. As illustrated in Fig. 1b, the artificial throat has the integrated functions of emitting and detecting sounds. When an AC voltage is applied on the device, the periodic joule heat will cause the expansion of air, resulting in sound waves. When a low bias voltage is applied on the device, the vibration of throat cords will cause the change of the device's resistance, resulting in a fluctuation of the current. Therefore, the devices can work as sound source and detector at the same time. The working principle of the LIG artificial throat is shown in Fig. 1c. A cough, hum or scream will cause the vibration of throat cords, which can be detected by the LIG artificial throat, and then LIG artificial throat will generate controllable sounds accordingly. Therefore, the LIG artificial throat can realize the conversion from meaningless sounds to controllable and predesigned sounds.

The photograph of LIG generated at different laser power $P$ ranging from 20 to $350 \mathrm{~mW}$ is shown in Fig. 1d. The most 

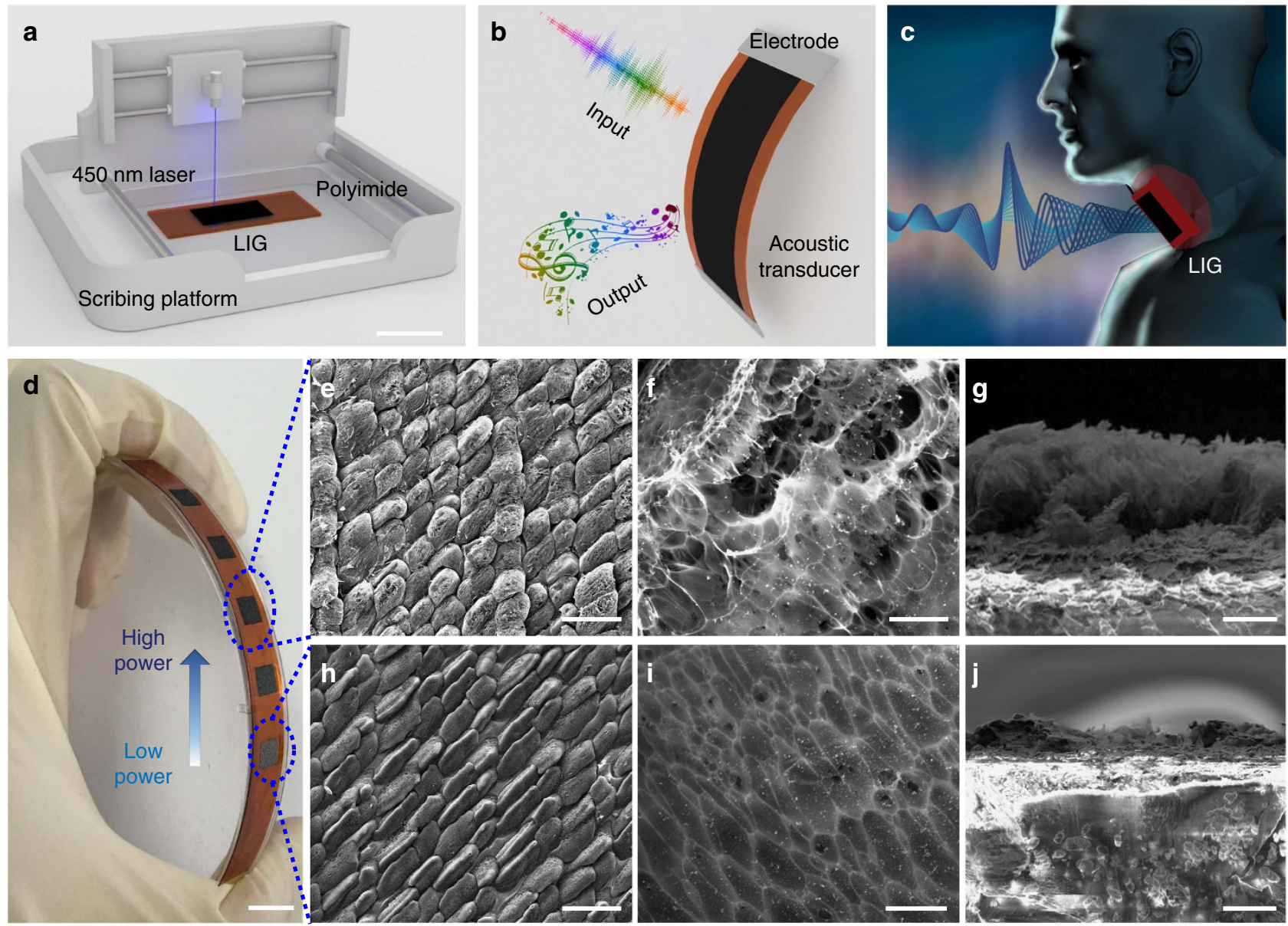

Figure 1 | Schematic illustration of the fabrication process and the morphology of LIG. (a) One-step fabrication process of LIG. PI is directly converted into LIG by the irradiation of the $450 \mathrm{~nm}$ laser. Scale bar, $2.5 \mathrm{~cm}$. (b) LIG has the ability of emitting and detecting sound in one device. (c) The artificial throat can detect the movement of throat and generate controllable sound, respectively. (d) Six LIG samples produced by $450 \mathrm{~nm}$ laser with different power ranging from 20 to $350 \mathrm{~mW}$. Scale bar, $5 \mathrm{~mm}$. (e) The morphology of LIG sample produced at $290 \mathrm{~mW}$ under scanning electron microscopy. Scale bar, $150 \mu \mathrm{m}$. (f) The morphology of LIG sample produced at $290 \mathrm{~mW}$ under high magnification. Scale bar, $5 \mu \mathrm{m}$. (g) Cross-sectional view of LIG sample produced at $290 \mathrm{~mW}$. Scale bar, $12.5 \mu \mathrm{m}$. (h) The morphology of LIG sample produced at $125 \mathrm{~mW}$ under scanning electron microscopy. Scale bar, $150 \mu \mathrm{m}$. (i) The morphology of LIG sample produced at $125 \mathrm{~mW}$ under high magnification. Scale bar, $5 \mu \mathrm{m}$. (j) Cross-sectional view of LIG sample produced at $125 \mathrm{~mW}$. Scale bar, $12.5 \mu \mathrm{m}$.

bottom LIG is irradiated at $P=20 \mathrm{~mW}$ and no obvious LIG is observed (as shown in Supplementary Fig. 3). The second one and the fourth one from the bottom, which are generated at $P=125 \mathrm{~mW}$ and $P=290 \mathrm{~mW}$, respectively, are chosen to show their scanning electron microscope images because of their typical morphologies. As shown in Fig. 1e-j, it can be noticed that ridgy lines are formed orderly along the scanning trace of laser from up to down. The line width is around $100 \mu \mathrm{m}$, which is similar to the focus spot size of the laser. Morphological differences can be clearly observed with the increasing of laser power. More pores are produced at $P=290 \mathrm{~mW}$. From the highmagnification image, the differences between the structures become more distinctive. A thin layer of carbon sheet with porous polygon appears at $P=125 \mathrm{~mW}$ and more porous and irregular structure is produced at $P=290 \mathrm{~mW}$, because local high temperature during laser heating conduct the pyrolysis of $\mathrm{P}^{38,39}$, breaking the $\mathrm{C}-\mathrm{O}, \mathrm{C}=\mathrm{O}$ and $\mathrm{N}-\mathrm{C}$ bonds, and causing the venting of some carbonaceous and nitric gases. From the side view, we can notice that the thickness increases from $\sim 10$ to $\sim 40 \mu \mathrm{m}$ at $P=125 \mathrm{~mW}$ and $P=290 \mathrm{~mW}$, respectively. The structure generated at $350 \mathrm{~mW}$ (Supplementary Fig. 4) becomes more porous and thicker.
The Raman spectrums of PI film and samples generated at $P=125 \mathrm{~mW}$ and $P=350 \mathrm{~mW}$ are performed for further investigation (Supplementary Fig. 5). The spectrums obtained at $P=125 \mathrm{~mW}$ and $P=350 \mathrm{~mW}$ show the similar characteristics with a $D, G$ and $2 D$ peak at $1,350,1,580$ and $2,700 \mathrm{~cm}^{-1}$ respectively. The Raman spectrum is clearly different from amorphous carbon, proving the existence of randomly graphene stacks. With the increasing of the laser power, the intensity of $D$ peak increases, indicating that high-power laser makes the structure more defective and disordered. Moreover, the highresolution transmission electron microscope image presents the lattice fringes with an interspace of $\sim 3.4 \AA$, corresponding to the interplanar spacing of (002) plane in graphic materials (Supplementary Fig. 6).

LIG artificial throat working as a sound source. Four samples of LIG artificial throats, which are produced at different laser powers, are used to test the performance of emitting sound. The area of the LIG is around $1 \times 2 \mathrm{~cm}^{2}$. The laser powers are 125 , 200, 290 and $350 \mathrm{~mW}$. The average thickness of LIG is 8,22 , 38 and $60 \mu \mathrm{m}$. The LIG artificial throat is clamped under a 
a

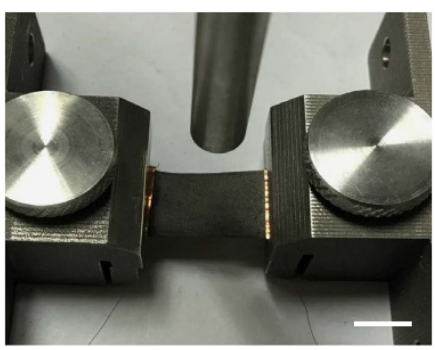

d

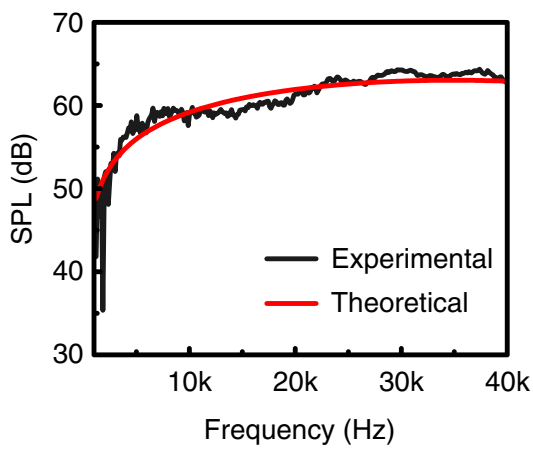

b

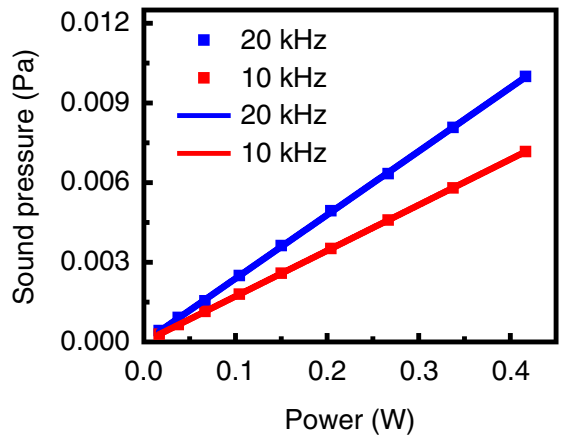

e

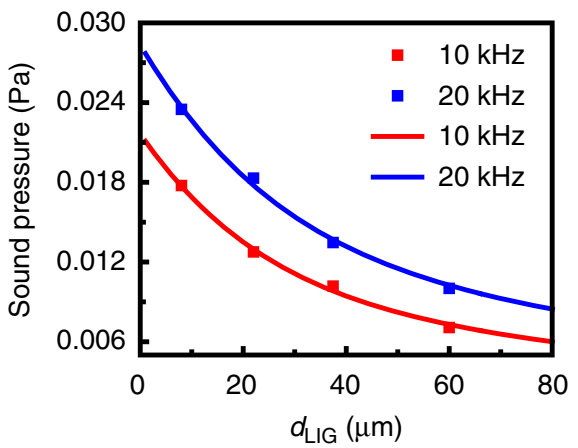

c

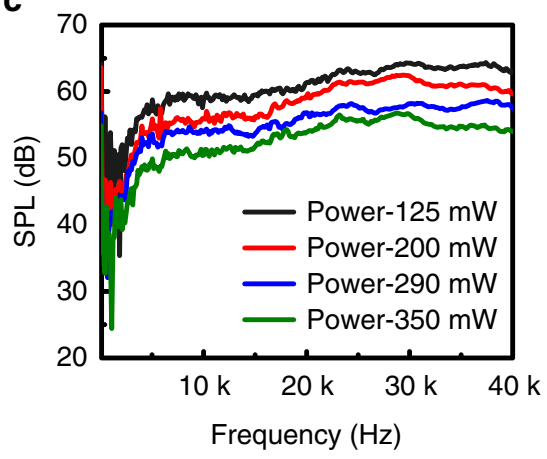

f

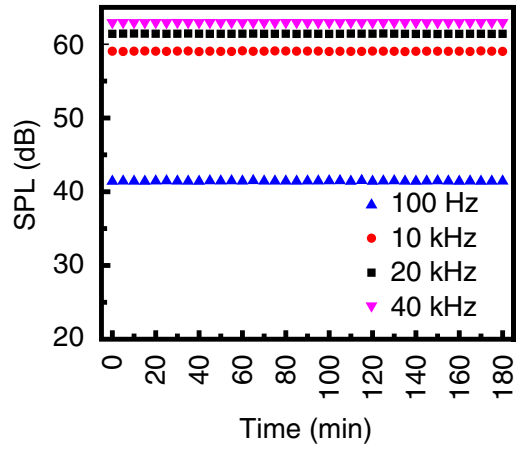

Figure 2 | The performance of emitting sound. (a) The LIG is clamped under a commercial microphone to test the performance of emitting sound. Scale bar, $1 \mathrm{~cm}$. (b) The plot of the SP versus the input power at 10 and $20 \mathrm{kHz}$. The square is the experimental result and the line is the theoretical result. (c) The output SPL versus the frequency of LIG generated by the laser with different power. The four curves are normalized with the input power of $1 \mathrm{~W}$. (d) The SPL versus the frequency showing that the model agrees well with experimental results. (e) The plot of the SP versus the thickness of LIG at 10 and $20 \mathrm{kHz}$. The square is the experimental result and the line is the theoretical result. (f) The stability of output SPL over time.

commercial microphone as shown in Fig. 2a. The distance between LIG and microphone is $2.5 \mathrm{~cm}$ (see the Methods section for details). As shown in Fig. 2b, the output SP of LIG (generated under $125 \mathrm{~mW}$ ) increases with the input power and the LIG has a higher efficiency at $20 \mathrm{kHz}$. The fitting line shows that the SP has a linear relation with the input power. The relationships between SPL and the frequency of different samples are demonstrated in Fig. 2c. The experimental results are normalized with the same power $(1 \mathrm{~W})$. It is noticed that the SPL becomes lower with the increasing of laser power and the thickness of LIG. A similar phenomenon was found in the work by Tian et al. ${ }^{6}$.

The theoretical model of thermal acoustic is first built by Arnold and Crandall ${ }^{40}$. In recent years, a new model of the thermal acoustic effect is proposed based on the energy conservation $^{41}$, which makes it easy to analyse and calculate. The core of thermal acoustic is the propagation of the heat. SP is decided by the thermal energy diffused into air: ${ }^{42}$

$$
p_{\mathrm{rms}}=\frac{m_{\mathrm{air}} \cdot f}{2 \sqrt{2} C_{\mathrm{p}} T_{0} r} \cdot \dot{Q}_{\mathrm{air}}
$$

where $m_{\text {air }}$ is the molecular weight of air, $f$ is the frequency of the acoustic, $Q_{\text {air }}$ is the thermal energy diffused into air, $C_{\mathrm{p}}$ is the heat capacity at constant pressure, $T_{0}$ is the room temperature and $r$ is the measuring distance from the source.

The thermal energy is calculated as: ${ }^{41}$

$$
\dot{Q}_{\text {air }}=\frac{2+2 \sqrt{2} \xi+2 \xi^{2}+\sqrt{2} \xi^{3}}{4 \times\left(1+\sqrt{2} \xi+\xi^{2}\right)^{2}} \times \frac{P_{\mathrm{e}}}{1+\chi}
$$

where

$$
\xi=\sqrt{\frac{\pi}{2}} \times \frac{\rho_{s} C_{s} d_{f} f^{0.5}}{\sqrt{\rho_{\mathrm{air}} C_{\mathrm{v}, \mathrm{air}} k_{\mathrm{air}}}+\sqrt{\rho_{\mathrm{PI}} C_{\mathrm{V}, \mathrm{PI}} k_{\mathrm{PI}}}}
$$

$\rho, C_{\mathrm{v}}$ and $k$ are the density, heat capacity at constant pressure and conductivity of each material, respectively. $d_{\mathrm{s}}$ is the thickness of the LIG and $P_{\mathrm{e}}$ is the input power. From equation (2), we can get the SPL as a function of frequency and the thickness of LIG.

Figure $2 \mathrm{~d}$ is the comparison of theoretical curve and the experimental results. The experimental analysis matches well with the theory model. Figure $2 \mathrm{e}$ shows the experimental data and theoretical curve as a function of the thickness of the LIG under the frequency of 10 and $20 \mathrm{kHz}$. The four samples with the thickness of $8,22,38$ and $60 \mu \mathrm{m}$ would produce the sounds with the SP of $0.01775,0.01276,0.01019$ and $0.00706 \mathrm{~Pa}$ at the frequency of $10 \mathrm{kHz}$ and $0.0235,0.0183,0.01346$ and $0.01 \mathrm{~Pa}$ at the frequency of $20 \mathrm{kHz}$, respectively. The theoretical curve matches well with the experimental results. As the decrease of the thickness, the leakage of thermal energy will be reduced and more thermal energy will be propagated into the air, implying that a thinner LIG will generate a higher SP. The experimental data fit well with the theoretical model. The working temperature is tested by a thermal infrared camera (Fluke Ti 200). The working temperature are $23.7^{\circ} \mathrm{C}$ with $0.4 \mathrm{~W}$ input power and below $29^{\circ} \mathrm{C}$ even with $1.225 \mathrm{~W}$ input power, which are acceptable when attached to the skin (Supplementary Fig. 7). The SPL is measured for a long time to investigate the stability of LIG. SPL at $100 \mathrm{~Hz}$, $10 \mathrm{kHz}, 20 \mathrm{kHz}$ and $40 \mathrm{kHz}$ are shown in Fig. 2f. There are no signs of degradation or changes in the device performance in $3 \mathrm{~h}$.

LIG artificial throat working as a sound detector. Except for emitting sound, the LIG artificial throat also has excellent responses when detecting sound. PI with different thickness will have different performance of recognition. A $25 \mu \mathrm{m}$-thick PI is 
a

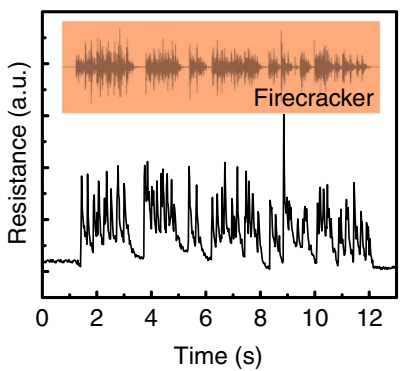

d

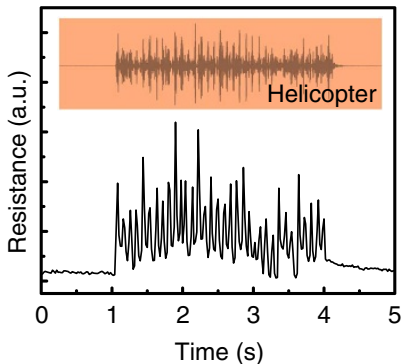

b

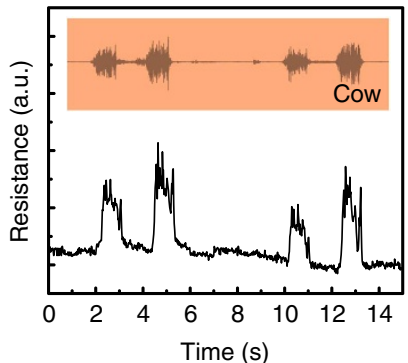

e

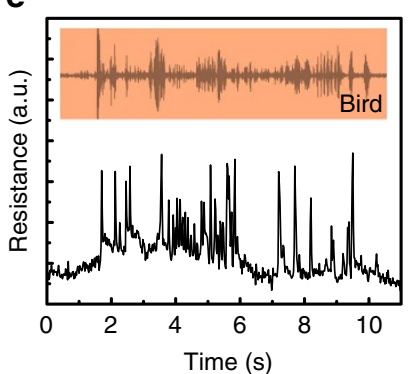

c

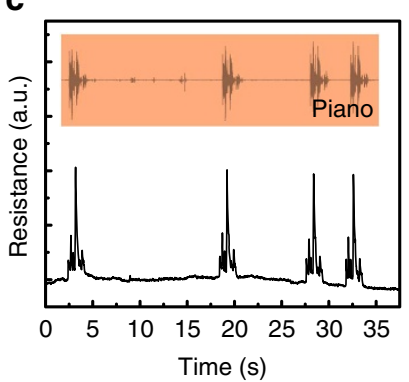

f

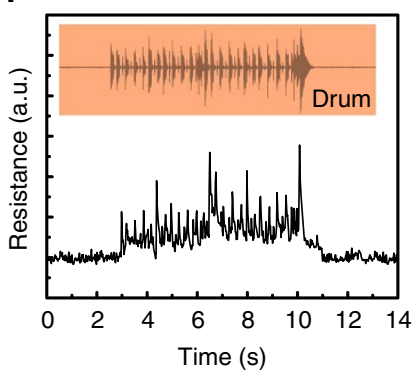

Figure 3 | Responses towards different audios from a loudspeaker. The LIG is placed $3 \mathrm{~cm}$ away from the loudspeaker. The orange insets above indicate the sound wave profiles of the original audios. Relative resistance changes show almost synchronous response to profiles of the original audios when the loudspeaker plays the audio of (a) firecrackers, (b) a cow, (c) a piano, (d) a helicopter, (e) a bird and (f) a drum.

chosen to generate LIG because of the obvious resistance change compared to the PI with the thickness of 75 and $180 \mu \mathrm{m}$ (Supplementary Fig. 8). The sensitivity is high enough to detect SP produced by a loudspeaker. The $25 \mu \mathrm{m}$ PI-LIG is fixed by two clamps free-standingly and the loudspeaker is placed $3 \mathrm{~cm}$ away from the artificial throat. The audio tests with six kinds of audios including firecracker, cow, piano, helicopter, bird and drum are performed. The results are shown in Fig. 3. Although the sampling frequency of our artificial throat is $100 \mathrm{~Hz}$, which is far lower than the frequency of sound, we can still notice that the responses of the transducer are well synchronous to these original audio signals (orange parts). Especially, the characteristic peaks are retained and reflected with high fidelity. Besides, the volume of loudspeaker has a great effect on the amplitude of the signal. With the volume increases, the vibration will be strengthened, causing a more obvious change of resistance (Supplementary Fig. 8). The film is clamped and bent under the force (Supplementary Fig. 9). The curvature radius is $1.27 \mathrm{~cm}$ and the stress is $0.07 \mathrm{~N}$. The bending degree is higher than the vibration degree, which is caused when speaking. The testing results after 0 cycle, 1,000 cycles and 10,000 cycles are shown in Supplementary Fig. 9. We can see the device shows excellent durability under high strain.

After identifying some kinds of audio clearly, LIG throat is used to detect the vibration of throat cords. As shown in Fig. 4a, the tester makes two successive coughs, hums and screams, and then the tester does swallowing and nod actions in two times. The repeatability of the detection is excellent according to the two-time successive testing. Besides, the swallowing and the nod can cause the muscle movement, which can also result in the change of the resistance. Fortunately, the waveforms of these kinds of muscle movements also have recognizable characteristics. Different movement has its unique characteristic waveform as shown in Fig. 4a; thus, we can get the useful waveforms by relying on the pattern recognition and machine learning. The interference by some other activities can be recognized and eliminated by training many times in advance. Then, the tester makes the hums with four different tones as shown in Fig. $4 \mathrm{~b}$; we can see that different tones also have different responses, increasing the variety of the 'language' of mute persons. Especially, the hum tone 2 is same with the hum in Fig. $4 \mathrm{a}$. Furthermore, as shown in Fig. 4c, the resistance increases as the sound intensity increases, resulting from the increase of the mechanical vibration of throat cords.

When the device is attached on the throat, it can detect both SP and throat vibration. An experiment is performed to compare whether the mechanical vibration of throat cords or the SP contributes most to the relative resistance change of the LIG artificial throat. The LIG is attached on the throat of the tester and the tester makes some hums with different volumes, and these sounds are recorded and played by a loudspeaker. The LIG then is placed $3 \mathrm{~cm}$ away from the loudspeaker, to acquire the signal induced by pure SP. According to Supplementary Fig. 10, the relative resistance change of the device placed $3 \mathrm{~cm}$ away from the loudspeaker is only $0.5 \%$ when the loudspeaker plays a sound of $90 \mathrm{~dB}$, which means the pure SPL of $90 \mathrm{~dB}$ will cause a $0.5 \%$ relative resistance change. However, the relative resistance change of the device attached on the throat can be $8.2 \%$ when a person makes a hum of $90 \mathrm{~dB}$. The relative resistance change of the device attached on the throat is 16 times larger than that caused by pure SP, which means the vibration of throat cords contributes more than the SP.

Besides, the LIG artificial throat also has the capability of voice recognition. As shown in Supplementary Fig. 11, some words including 'Graphene', 'Material' and 'Industry' are pronounced by different testers including an elder, a boy, a man and a woman, respectively. The wave curves of different words in the time domain show apparently different characteristics, which is helpful to distinguish different words. Besides, the wave curves of a same word pronounced by different persons show similar but different characteristics, which can be a key factor for identity authentication by voice recognition. Furthermore, a long sentence, 'Graphene is a carbon-based material with huge potential for industry', is spoken repeatedly by a woman for five times. The artificial throat shows excellent repeatability and reliability to 

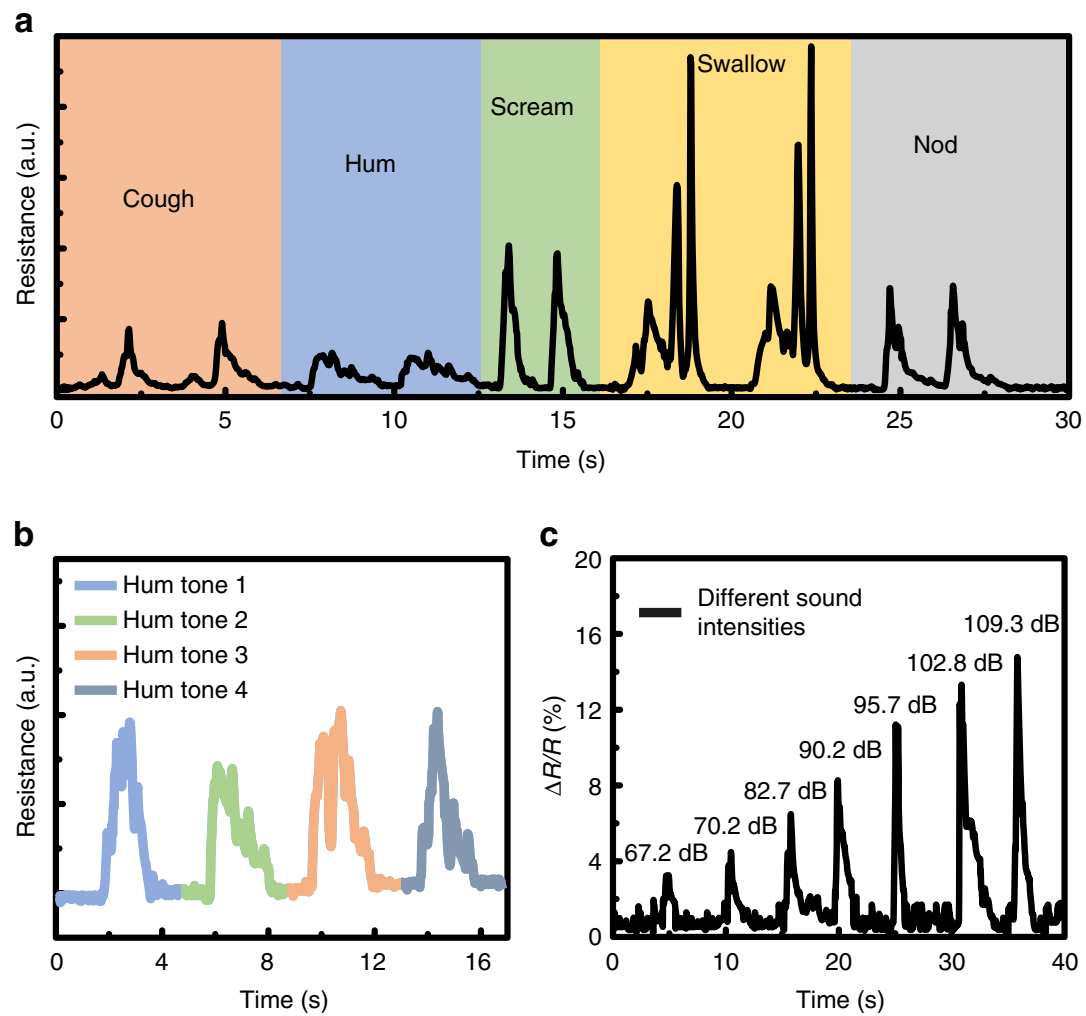

Figure 4 | Responses towards different kinds of throat vibrations. (a) The LIG's resistance changes towards the throat vibrations of the tester who makes two successive coughs, hums, screams, swallowing and nods. (b) The LIG's resistance change caused by four different kinds of hum tones and the hum tone 2 is same with the hum in $\mathbf{a}$. (c) The relative resistance change of LIG increases with the increase of the sound intensities of the hum.

work as an acoustic detector. Especially, from the magnified image of the sentence, we can notice that the three words, 'graphene', 'material' and 'industry', are almost identical to the individual pronunciation by the woman. Compared with some other nanomaterial-based acoustic detectors ${ }^{17-22}$, our device demonstrates superior predominance in voice recognition because of its excellent repeatability and reliability.

Working as sound source and sound detector simultaneously. The LIG artificial throat developed in this work has great potential to bring a revolution in the field of acoustic. As we know, most mute persons are born deaf and they cannot speak words. However, their throat cords can vibrate and they are capable of making noises in their own ways, which are meaningless to the normal. The intelligent LIG artificial throat is developed to transform the meaningless noise into controllable and understandable sound signals. The testers need to be trained first. When they produced a specific cough, hum or scream, we told them the corresponding meaning by gesture language. They can be accustomed to the sound intensity by multi-time repeated training. The training process is similar with the process of importing the fingerprint into iPhone. Then, these waveforms can be recognized by pattern recognition and machine learning. Besides, we set some threshold ranges in advance. The software can recognize the sound intensity when it lies on the threshold range. The accuracy of recognition can be guaranteed by the above two ways. After the training process, the different volume, frequency and last time of hum, scream or cough can be converted into specific meanings. Here we simply demonstrated the conversion from three kinds of different hums to three controllable sounds. The working principle of LIG artificial throat is shown in Fig. 5a (see the Methods section for details). It works as a sound detector at first, then the tester makes three kinds of hums to imitate a mute person and the resistance of the transducer will be changed by the throat cords vibration correspondingly. A microcontroller is used to detect the resistance change of the transducer and realize the hum judgement. After that, the transducer begins to work as a sound source and corresponding sound signal is generated. The LIG artificial throat is attached on the throat of a tester as Fig. 5b shows.

As shown in Fig. 5c, a high-volume hum, a low-volume hum and an elongated hum are pronounced twice by the tester. Correspondingly, a high-volume $10 \mathrm{kHz}$, a low-volume $10 \mathrm{kHz}$ and a low-volume $5 \mathrm{kHz}$ sound signal are produced. The output sound frequency is twice the input electric signal; thus, a DC bias is added to generate the fundamental tone $\mathrm{e}^{11}$. The high-volume $10 \mathrm{kHz}$, the low-volume $10 \mathrm{kHz}$ and the low-volume $5 \mathrm{kHz}$ sound are generated by $10 \mathrm{~V} \mathrm{DC}+10 \mathrm{~V} 10 \mathrm{kHz}$ AC, $5 \mathrm{~V} \mathrm{DC}+5 \mathrm{~V}$ $10 \mathrm{kHz} A C$ and $5 \mathrm{~V} \mathrm{DC}+5 \mathrm{~V} 5 \mathrm{kHz}$ AC, respectively (Supplementary Movie 1). From the magnified images (Fig. $5 \mathrm{~d}-\mathrm{f}$ ), the amplitude and the frequency of the output sound signals are shown as we expected. Finally, an intelligent LIG artificial throat is demonstrated to convert meaningless noises into useful sound signals with controllable frequencies and volumes. It will be possible for mute persons to express themselves with the assistance of LIG artificial throat.

\section{Discussion}

In summary, a one-step fabricated wearable artificial throat based on LIG has been developed. The low-power laser with the wavelength of $450 \mathrm{~nm}$ can induce the conversion from PI to LIG. The LIG realize the functional integration of emitting and detecting sound on a single device because of its superior thermoacoustic and piezoresistive properties. As a sound source, the SPL of the LIG artificial throat has been demonstrated from $100 \mathrm{~Hz}$ to $40 \mathrm{kHz}$. The thickness of LIG will have an obvious 
a

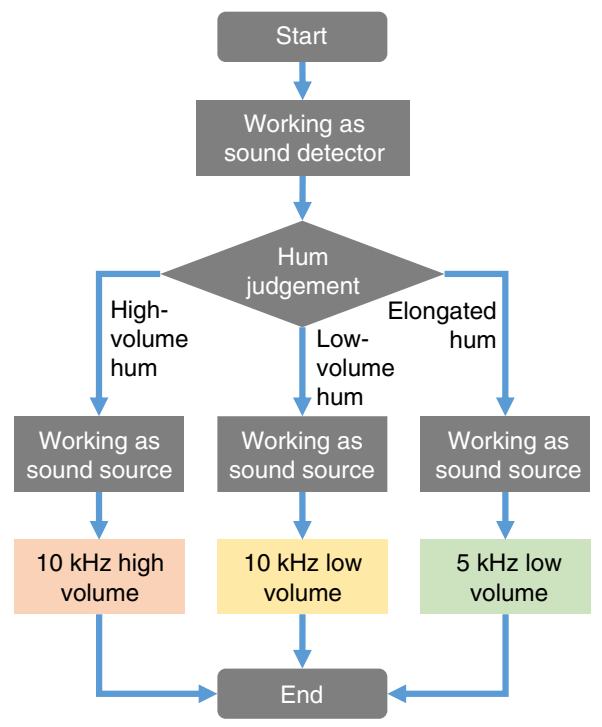

b

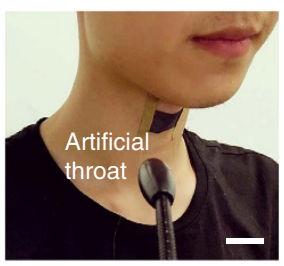

d

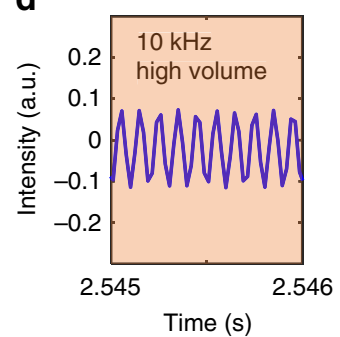

c

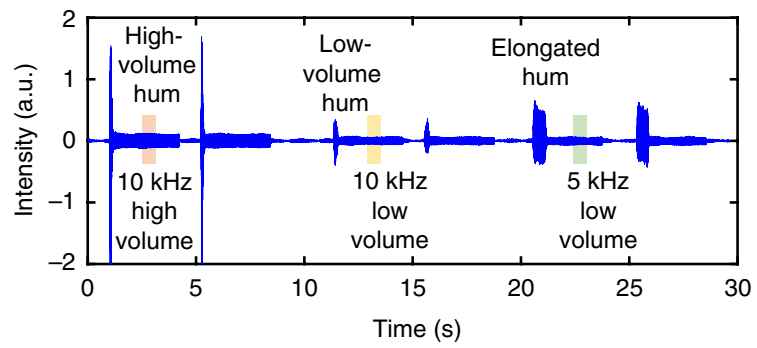

e

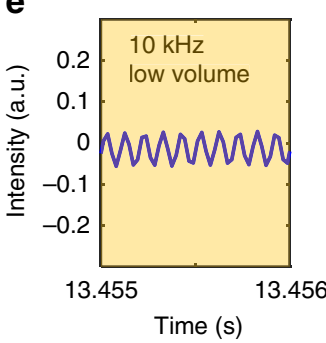

f

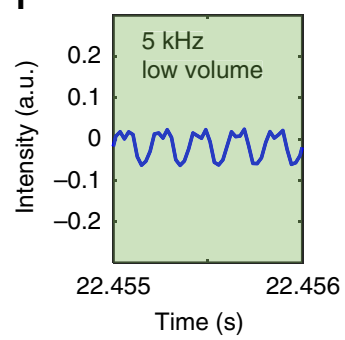

Figure 5 | An intelligent LIG artificial throat. (a) The working procedure of the artificial throat. (b) The tester wearing the LIG artificial throat. Scale bar, $1 \mathrm{~cm}$. (c) high-volume, low-volume and elongated tone hum are detected by LIG throat and converted into high-volume $10 \mathrm{kHz}$, low-volume $10 \mathrm{kHz}$ and low-volume $5 \mathrm{kHz}$ sound, respectively. (d) The magnified wave of high-volume $10 \mathrm{kHz}$ sound. (e) The magnified wave of low-volume $10 \mathrm{kHz}$ sound. (f) The magnified wave of low-volume $5 \mathrm{kHz}$ sound.

influence on SPL according to our theory and a thinner LIG will generate sound with higher SPL. The LIG artificial throat has a relatively broad frequency spectrum because of resonance-free oscillations of the sound sources. Besides, as a sound detector, the LIG artificial throat can capture the mechanical vibration of throat cords with a fine repetition. It can clearly differentiate the characteristics of cough, hum and scream with different tones and volumes according to their unique waveforms. Besides, it also has the capability of voice recognition because of its outstanding mechanical properties. The intelligent LIG artificial throat will significantly assist for disabled person. The LIG artificial throat can generate volume and frequency controllable sound by detecting different kinds of imitative hum of the tester and realize the conversion from meaningless hum to controllable sound, which has significantly practical potentials. Furthermore, the one-step fabricated wearable LIG artificial throat will open widely practical applications in voice control, wearable electronics and many other areas due to its high sensitivity, excellent repetition, good flexibility and simple fabrication process.

\section{Methods}

Fabrication of artificial throat. A custom-designed platform equipped with a laser diode (OSRAM) with wavelength of $450 \mathrm{~nm}$ was used to convert PI into LIG. Figure la shows the schematic diagram of the mask-free process. The movement of laser was controlled by two stepper motors in $X-Y$ direction. The beam size of laser and the minimum displacement of stepper motor are $100 \mu \mathrm{m}$. The scanning speed of laser is $8.5 \mathrm{~mm} \mathrm{~s}^{-1}$ and the maximum power is $500 \mathrm{~mW}$. Commercial PI films (Kapon) with thickness of 25, 75, 125 and $180 \mu \mathrm{m}$ were used as the substrate without any further treatment. After the laser irradiation, the LIG artificial throats are wired out by copper wire by using silver paste.

Characterization. The surface morphology of the LIG is observed by a Quanta FEG 450 scanning electron microscope (FEI Inc.). Raman spectroscopy is performed using a laser with a wavelength of $532 \mathrm{~nm}$ (HORIBA Inc.). Transmission electron microscopic images are taken by JEM2100F (JEOL Inc.).

Testing of artificial throat. The sound emitting testing platform consisted of a standard microphone and a dynamic signal analyser. A $1 / 4$ inch standard microphone (Earthworks M50) was chosen to measure the SPL of the LIG artificial throat. The microphone has a very flat frequency response within $40 \mathrm{kHz}$ and a high sensitivity of $31 \mathrm{mV} \mathrm{Pa}^{-1}$. The dynamic signal analyser (Agilent $35670 \mathrm{~A}$ ) was used to generate swept-frequency signals from $100 \mathrm{~Hz}$ to $40 \mathrm{kHz}$, to drive the LIG artificial throat and to record the value of the SPL of it. The sound-detecting testing platform consisted of a commercial loudspeaker and a digital multimeter. LIG artificial throat was placed $3 \mathrm{~cm}$ away from the loudspeaker. The digital multimeter (ROGOL DM3068) was used to the relationship between the resistance and the time.

Generating and detecting sound in a single device. The LIG artificial throat was attached on the throat of a tester. The LIG artificial throat worked in two modes: detecting mode and emitting mode. During the detecting mode, a microcontroller was used to detect the amplitude and last time of the meaningless hums. When the amplitude or last time exceeded the thresholds, the microcontroller would stop detecting and the digital function generator would be applied on the LIG artificial throat for $3 \mathrm{~s}$. After $3 \mathrm{~s}$, the microcontroller closed the digital function generator by a relay and LIG artificial throat works in detecting mode again. Different digital function generators would be applied on the LIG artificial throat respectively according to the amplitude and last time of the meaningless noise.

Data availability. The data that support the findings of this study are available from the corresponding author upon request.

\section{References}

1. Jain, M., Puri, S. \& Unishree, S. Eyeball motion controlled wheelchair using IR sensors. World Acad. Sci. Eng. Technol. Int. J. Comput. Electr. Autom. Control Inf. Eng. 9, 906-909 (2015).

2. Thakur, B. \& Kulshrestha, K. in Proceedings of IEEE International Conference on Computational Intelligence and Computing Research (ICCIC) 1-6 (Coimbatore, India, 18-20 December 2014).

3. Brown, L. F. \& Carlson, D. L. Ultrasound transducer models for piezoelectric polymer films. IEEE Trans. Ultrason. Ferroelectr. Freq. Control 36, 313-318 (1989).

4. Marciniak, R. D. Unidirectional underwater-sound pressure-gradient transducer. IEEE Trans. Sonics Ultrason. 18, 89-94 (1971).

5. Meeks, S. W. \& Timme, R. W. Rare earth iron magnetostrictive underwater sound transducer. J. Acoust. Soc. Am. 62, 1158-1164 (1977).

6. Tian, H. et al. Graphene-on-paper sound source devices. ACS Nano 5, 4878-4885 (2011).

7. Suk, J. W., Kirk, K., Hao, Y., Hall, N. A. \& Ruoff, R. S. Thermoacoustic sound generation from monolayer graphene for transparent and flexible sound sources. Adv. Mater. 24, 6342-6347 (2012).

8. Tian, H. et al. Single-layer graphene sound-emitting devices: experiments and modeling. Nanoscale 4, 2272-2277 (2012).

9. Tian, H. et al. Static behavior of a graphene-based sound-emitting device. Nanoscale 4, 3345-3349 (2012). 
10. Tian, H. et al. A flexible, transparent and ultrathin single-layer graphene earphone. RSC Adv. 5, 17366-17371 (2015).

11. Tian, H. et al. Graphene earphones: entertainment for both humans and animals. ACS Nano 8, 5883-5890 (2014).

12. Tian, H., Yang, Y., Xie, D., Ge, J. \& Ren, T.-L. A reduced graphene oxide soundemitting device: a new use for Joule heating. RSC Adv. 3, 17672-17676 (2013).

13. Xiao, L. et al. Flexible, stretchable, transparent carbon nanotube thin film loudspeakers. Nano Lett. 8, 4539-4545 (2008).

14. Mason, B. J., Chang, S., Chen, J., Cronin, S. B. \& Bushmaker, A. W. Thermoacoustic transduction in individual suspended carbon nanotubes. ACS Nano 9, 5372-5376 (2015).

15. Tian, H. et al. Flexible, ultrathin, and transparent sound-emitting devices using silver nanowires film. Appl. Phys. Lett. 99, 253507 (2011).

16. Tian, H. et al. Transparent, flexible, ultrathin sound source devices using indium tin oxide films. Appl. Phys. Lett. 99, 043503 (2011).

17. Wang, Y. et al. Wearable and highly sensitive graphene strain sensors for human motion monitoring. Adv. Funct. Mater. 24, 4666-4670 (2014).

18. Tang, Y. et al. Highly stretchable and ultrasensitive strain sensor based on reduced graphene oxide microtubes-elastomer composite. ACS Appl. Mater. Interfaces 7, 27432-27439 (2015).

19. Wang, Y. et al. Ultra-sensitive graphene strain sensor for sound signal acquisition and recognition. Nano Res. 8, 1627-1636 (2015).

20. Wang, C. et al. Carbonized silk fabric for ultrastretchable, highly sensitive, and wearable strain sensors. Adv. Mater. 28, 6640-6648 (2016).

21. Gong, S. et al. Highly stretchy black gold e-skin nanopatches as highly sensitive wearable biomedical sensors. Adv. Electron. Mater. 1, 1400063 (2015).

22. Park, J. J., Hyun, W. J., Mun, S. C., Park, Y. T. \& Park, O. O. Highly stretchable and wearable graphene strain sensors with controllable sensitivity for human motion monitoring. ACS Appl. Mater. Interfaces 7, 6317-6324 (2015).

23. Cheng, Y., Wang, R., Sun, J. \& Gao, L. A stretchable and highly sensitive graphene-based fiber for sensing tensile strain, bending, and torsion. Adv. Mater. 27, 7365-7371 (2015).

24. Tian, H. et al. Scalable fabrication of high-performance and flexible graphene strain sensors. Nanoscale 6, 699-705 (2014).

25. El-Kady, M. F., Strong, V., Dubin, S. \& Kaner, R. B. Laser scribing of highperformance and flexible graphene-based electrochemical capacitors. Science 335, 1326-1330 (2012).

26. El-Kady, M. F. \& Kaner, R. B. Direct laser writing of graphene electronics. ACS Nano 8, 8725-8729 (2014).

27. Tian, H. et al. Cost-effective, transfer-free, flexible resistive random access memory using laser-scribed reduced graphene oxide patterning technology. Nano Lett. 14, 3214-3219 (2014).

28. Tian, H. et al. Wafer-scale integration of graphene-based electronic, optoelectronic and electroacoustic devices. Sci. Rep. 4, 3598 (2014).

29. Gao, W. et al. Direct laser writing of micro-supercapacitors on hydrated graphite oxide films. Nat. Nanotechnol. 6, 496-500 (2011).

30. El-Kady, M. F. \& Kaner, R. B. Scalable fabrication of high-power graphene micro-supercapacitors for flexible and on-chip energy storage. Nat. Commun. 4, 1475 (2013).

31. Lin, J. et al. Laser-induced porous graphene films from commercial polymers. Nat. Commun. 5, 5714 (2014)

32. In, J. B. et al. Facile fabrication of flexible all solid-state micro-supercapacitor by direct laser writing of porous carbon in polyimide. Carbon 83, 144-151 (2015).

33. Luo, S., Hoang, P. T. \& Liu, T. Direct laser writing for creating porous graphitic structures and their use for flexible and highly sensitive sensor and sensor arrays. Carbon 96, 522-531 (2016).

34. Rahimi, R., Ochoa, M., Yu, W. \& Ziaie, B. Highly stretchable and sensitive unidirectional strain sensor via laser carbonization. ACS Appl. Mater. Interfaces 7, 4463-4470 (2015)

35. Desilets, C. S., Fraser, J. D. \& Kino, G. S. The design of efficient broadband piezoelectric transducers. IEEE Trans. Sonics Ultrason. 25, 115-125 (1978).
36. Kossoff, G. The effects of backing and matching on the performance of piezoelectric ceramic transducers. IEEE Trans. Sonics Ultrason. 13, 20-30 (1966).

37. Manthey, W., Kroemer, N. \& Magori, V. Ultrasonic transducers and transducer arrays for applications in air. Meas. Sci. Technol. 3, 249-261 (1992).

38. Wynn, G. H. \& Fountain, A. W. Development and characterization of electrochemical devices using ultraviolet laser induced carbonization of polyimide films. J. Electrochem. Soc. 144, 3769-3772 (1997).

39. Brannon, J. H. \& Lankard, J. R. Pulsed CO2 laser etching of polyimide. Appl. Phys. Lett. 48, 1226-1228 (1986).

40. Arnold, H. D. \& Crandall, I. B. The thermophone as a precision source of sound. Phys. Rev. 10, 22-38 (1917).

41. Xie, Q. Y. et al. A point acoustic device based on aluminum nanowires. Nanoscale 8, 5516-5525 (2016).

42. Asadzadeh, S. S., Moosavi, A., Huynh, C. \& Saleki, O. Thermo acoustic study of carbon nanotubes in near and far field: theory, simulation, and experiment. J. Appl. Phys. 117, 095101 (2015).

\section{Acknowledgements}

This work was supported by National Natural Science Foundation (61574083 and 61434001), National Basic Research Program (2015CB352101), National Key Research and Development Program (2016YFA0200400), National Key Project of Science and Technology (2011ZX02403-002) and Special Fund for Agroscientific Research in the Public Interest (201303107) of China. We are thankful for the support of the Independent Research Program (2014Z01006) of Tsinghua University, and Advanced Sensor and Integrated System Lab of Tsinghua University Graduate School at Shenzhen under project number ZDSYS20140509172959969.

\section{Author contributions}

L.-Q.T. and H.T. discovered the procedures, designed most of the data and wrote the manuscript with equal contribution. Y.L. performed scanning electron microscopy and Raman characterizations. Z.-Y.J. conducted the modelling and theory. Y.P. performed transmission electron microscope characterizations. Y.-Q.C. and D.-Y.W. conducted some figures. X.-G.T., J.-C.Y. and N.-Q.D. provided valuable discussion. Finally, Y.Y. and T.-L.R. provided guidance to the research.

\section{Additional information}

Supplementary Information accompanies this paper at http://www.nature.com/ naturecommunications

Competing financial interests: The authors declare no competing financial interests.

Reprints and permission information is available online at http://npg.nature.com/ reprintsandpermissions/

How to cite this article: Tao, L.-Q. et al. An intelligent artificial throat with sound-sensing ability based on laser induced graphene. Nat. Commun. 8, 14579 doi: 10.1038/ncomms14579 (2017).

Publisher's note: Springer Nature remains neutral with regard to jurisdictional claims in published maps and institutional affiliations.

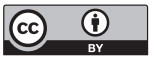

This work is licensed under a Creative Commons Attribution 4.0 International License. The images or other third party material in this article are included in the article's Creative Commons license, unless indicated otherwise in the credit line; if the material is not included under the Creative Commons license, users will need to obtain permission from the license holder to reproduce the material. To view a copy of this license, visit http://creativecommons.org/licenses/by/4.0/

C The Author(s) 2017 